\title{
Metal Oxide Sensors Applied to Industrial and \\ Consumer Applications: Examples and Requirements for Successful Implementation ${ }^{+}$
}

\author{
Debra J. Deininger \\ IDT, a Renesas Company, 2605 Trade Centre Ave, Suite C, Longmont, CO 80503, USA; \\ Debra.Deininger@idt.com; Tel.: +1-303-669-8194 \\ + Presented at the 8th GOSPEL Workshop. Gas Sensors Based on Semiconducting Metal Oxides: Basic \\ Understanding \& Application Fields, Ferrara, Italy, 20-21 June 2019.
}

Published: 19 June 2019

\begin{abstract}
Metal Oxide (MOx) sensors have been the subject of intense research and development over the years, and are widely used in industrial and commercial sensing applications. This talk will review the application of such gas sensors for industrial and consumer applications, with a focus on the requirements for successful implementation.

MOx sensors have several advantages over other competing sensor technologies in industrial and consumer applications. These sensors offer a highly reliable platform with long lifetimes, which is able to withstand temperature and humidity extremes without damage. Furthermore, MOx sensors can be produced with excellent economies of scale, especially when modern manufacturing methods are leveraged for silicon micro-hotplates.

The successful implementation of MOx sensors in industrial and consumer applications requires consideration of both the application and the technology. The most important items are discussed here.
\end{abstract}

\section{Application of MOx Sensors to Industrial and Commercial Applications-Keys to Success}

There are three key aspects to consider when matching MOx sensors to applications: (1) understanding the full application requirements, (2) designing the sensor, including selection of MOx materials and processing, and (3) developing the product, including the design of supporting electronics, calibration (if used) and algorithms to transduce the raw sensor signal (resistance) into useful information. The key to success is balancing the application requirements with both the benefits and limitations of the underlying technology. If the requirements and technology are well matched, then the end user / product is provided with useful and reliable information than benefits the person/product.

\section{Understanding the Application}

The most important key to success in the deployment of MOx sensors is always to understand the application. MOx sensors are fundamentally non-selective, so the first and most critical factors to understand are what gas(es) are expected to be in the environment and at what concentrations they should be detected, and equally important, what gas(es) are expected to be in the environment at concentrations that should not trigger a response. The environmental conditions, including temperature, humidity and barometric pressure should also be considered. The necessary information can be acquired from customer interactions, first-hand experience, literature review, or a combination of all of these sources. 
Another thing to consider is what the desired information output is, and how the end user or product will act upon the information. Is an absolute measurement of concentration required, or would relative concentration or rate of change be more useful? Will the sensor be used in a numeric display, or to trigger a warning or mechanical device? What are the response time, accuracy and precision requirements? Over-specification of requirements, or defining accuracy and precision more tightly than actually required should be avoided, as this will drive unnecessary system costs, while under-specification leads to disappointed or unhappy customers, which should also be equally avoided.

\section{MOx Materials Selection and Design}

Following a clear definition of the application and requirements, the MOx sensor design begins. There are a range of interesting materials and fabrication methods that have been promoted in the literature by commercial sensor developers. While sensitivity to the target gas(es) is required, there are some important additional criteria that also need to be established.

First, the sensors must be validated under the expected application conditions, including all gases and environmental variations uncovered in the application review. Sufficient quantities of sensors must be characterized under the expected application conditions to establish both sensor reproducibility and stability. In consumer and industrial applications, expectations of performance are measured in years, so particular attention to long-term stability and potential drift and failure modes is required.

The MOx material and deposition process, as well as the sensor substrate must be designed and characterized for reproducibility in high volume manufacturing. As gas testing is not a standard industrial process for most sensor and semiconductor manufacturers, particular attention should be focused on selection of final test method and conditions, including sensor calibration, if implemented.

\section{Product Development}

The final aspect of development to be considered is the development of a final product. While a stable and reliable MOx material and sensor is a necessary requirement for a good product, it is not sufficient to enable the final product.

The electronics used to control the MOx sensor heater, and to measure the resistance, should be considered, including the necessity for design of accurate heater control and the ability to measure large variations in resistance, depending on the MOx material used.

The sensor response will need to be converted from resistance to information, such as ppm, air quality level, or perhaps rate and magnitude of signal change if the sensor is used in a process control application. Methods of managing sensor aging and drift, coupled with the model used to convert between resistance and concentration, will greatly affect results.

\section{Example Implementations}

The oral presentation will cover two examples of sensor implantation in real world applications, air quality and breath measurements. The above factors will be discussed in the context of real-world data and decision making.

Funding: This research received no external funding.

Acknowledgments: The author thanks her employer, IDT, and the talented team of individuals working in the gas sensor development team for support.

Conflicts of Interest: The author declares no conflict of interest

(c) 2019 by the authors. Licensee MDPI, Basel, Switzerland. This article is an open access article distributed under the terms and conditions of the Creative Commons Attribution (CC BY) license (http://creativecommons.org/licenses/by/4.0/). 